\title{
Characterization of single-nucleotide polymorphisms in coding regions of human genes
}

Michele Cargill et al.

Nature Genet. 22, 231-238 (1999).

We inadvertently omitted Nila Shaw from our list of authors. The correct author list follows.

Michele Cargill, David Altshuler, James Ireland, Pamela Sklar, Kristin Ardlie, Nila Patil, Nila Shaw, Charles R. Lane, Esther P. Lim, Nilesh Kalyanaraman, James Nemesh, Liuda Ziaugra, Lisa Friedland, Alex Rolfe, Janet Warrington, Robert Lipshutz, George Q. Daley \& Eric S. Lander

\section{Griscelli disease maps to chromocome $15 q$ and is associated with mutations in the Myosin-Va gene}

Elodie Pastural et al.

Nature Genet. 16, 289-292 (1997).

One of the two Myosin Va mutations described, the Arg $\rightarrow$ Cys alteration at codon 1,246 ( $\rightarrow$ T transition at nt 3,736), was found to be a relatively common polymorphism. Several healthy individuals are homozygous carriers of the Cys 1246 allele. This fact was first pointed out to Nature Genetics by Jo Lambert, Jean Marie Naeyeart, Anne de Paepe, Rudy van Coster, Alina Ferster, Michele Song and Ludwine Messiaen of the University of Gent, Gent, Belgium. It was subsequently acknowledged by Pastural et al.

\section{Suppression of the novel growth inhibitor $\mathrm{p} 33^{\mathrm{ING} 1}$ promotes neoplastic transformation}

Igor Garkavtsev et al.

Nature Genet. 14, 415-420 (1996).

Due to a cloning error, the sequence reported for ING1 was incorrect. The error appears to have been a result of a compression introducing a frameshift and of the ING1 gene encoding several differentially spliced isoforms that contain a common $3^{\prime}$ exon, one of which is of a size very similar to that reported in the publication above. The original (ING1 $a^{\prime}$, previously called ING1) and the corrected (ING1a) sequences of the isoform of ING1 that was first reported are shown at right $(a)$. The sequence that we reported was frameshifted and truncated at the $5^{\prime}$ end, and we now know that it encodes a protein with a predicted mass of 46,751 rather than the predicted 33,253 daltons. A truncated ING1a message also encodes an expressed protein of 23,656 daltons that results from initiation at the first internal ATG in the conserved exon. An alternatively spliced isoform, ING1b, shares a common $3^{\prime}$ exon with ING1 $a$ and encodes a protein with a predicted mass of 31,843 daltons. We now refer to the proteins encoded by this gene as $\mathrm{p} 47^{\text {INGla }}$, p $33^{\text {ING1b }}$ and p24 ING1c due to their relative electrophoretic mobilities. These polypeptides are found in the majority of primary and established tissue culture cells examined, including those of fibroblast, epithelial and glial origin. A schematic diagram of the proteins encoded by the two major transcripts of ING1 are shown $(b)$. All three proteins contain a region with a high degree of homology to PHD fingers that are implicated in transcriptional regulation.

The complete, corrected nucleotide sequences for the cDNAs encoding ING1 a and - $b$ are available at accession numbers AF181849 (for ING1a, formerly listed as AF001954) and AF181850 at GenBank. We regret any inconvenience that may have resulted from the sequencing error. Expression constructs encoding fulllength ING1a, ING1b and ING1c proteins are available from Dongping Ma, Denise Lawless and Karl Riabowol, Department of Biochemistry and Molecular Biology, University of Calgary HSC, 3330 Hospital Dr. NW, Calgary, Alberta, Canada T2N 4N1. $a$

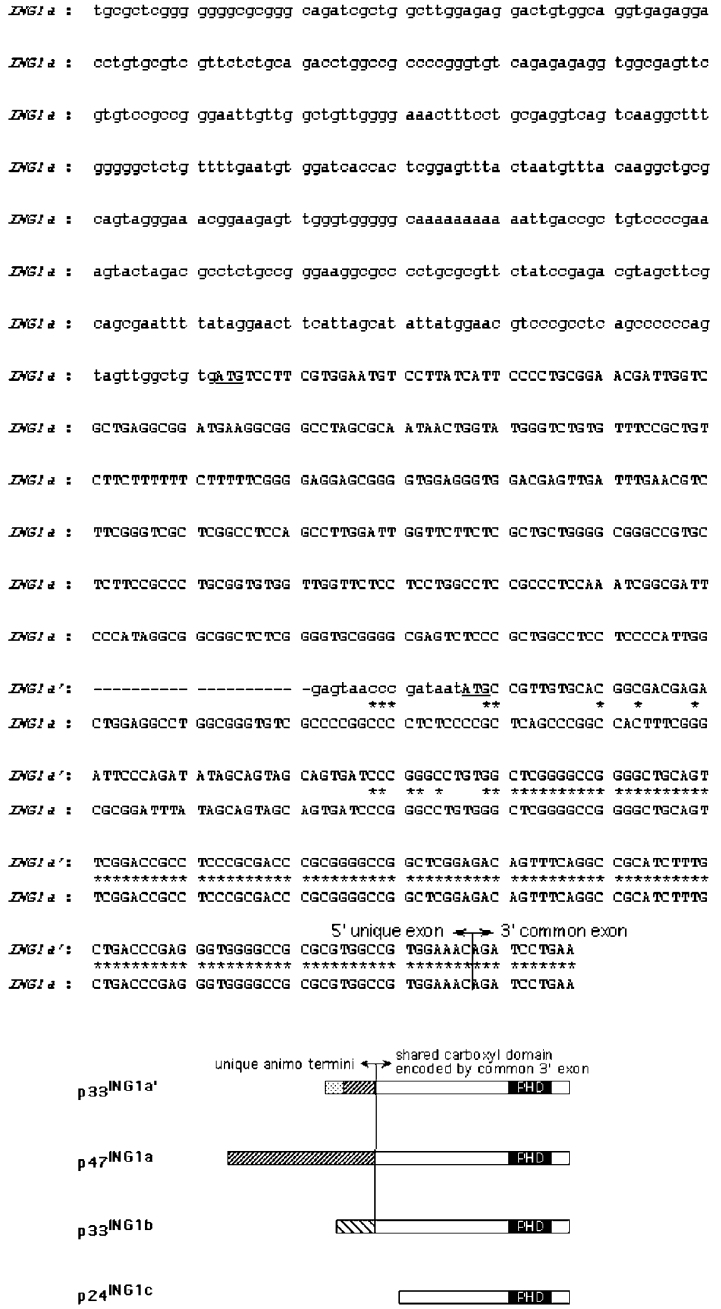

